\title{
Optical trapping at low numerical aperture
}

S. Stallinga
s.stallinga@tudelft.n

A theory of optical trapping at low Numerical Aperture (NA) is presented. The theory offers an analytical description of the competition between the stabilizing gradient and destabilizing scattering force. The trade-off can be characterized by a single dimensionless trapping parameter, which increases with bead radius to wavelength ratio $a / \lambda$ and refractive index contrast $m$ and decreases with NA. The gradient force dominates for small trapping parameters, the scattering force for large trapping parameters. The potential well depth, maximum forces and trap stiffness as a function of the three parameters $(a / \lambda, m, \mathrm{NA})$ can be mapped onto universal functions of the trapping parameter. These functions do not depend on any free parameter. The universal well depth and maximum force curves match with numerical results based on the exact multipole expansion of the optical trapping force. The paraxial limit of low NA is relevant for compact optical tweezers based on Optical Pickup Units known from optical data storage. [D0I: 10.2971/jeos.2011.11027]

Keywords: optical trapping, paraxial limit, gradient force, scattering force, compact optical tweezers

\section{INTRODUCTION}

Optical tweezers are now routinely used for trapping and manipulating beads, cells and other objects in the nano to microrange $[1,2]$. A good optical trap requires a focusing lens with a high Numerical Aperture (NA). Usually, a water immersion objective (NA around 1.25) or an oil immersion objective that is stopped down for focusing into a watery environment will do. This paper addresses the question if a high NA is absolutely necessary. Wei and Chiou [3] have shown optical trapping at NA $=0.85$. Is it possible to construct a stable optical trap at even lower NA? Will it work for any bead or object or just for a subset of possible objects? What are the parameter ranges (size, refractive index contrast) pertaining to these subsets? Is there in fact a lower limit to NA below which there can be no trapping at all? These questions will be answered in this paper by means of a fully analytical theory for describing the axial trap quality of low NA optical tweezers. The theory corresponds to the paraxial limit (low NA) of the exact theory of Mazzoli, Maia Neto and Nussenzveig $[4,5]$ and earlier workers $[6,7]$. This theory, experimentally tested and verified in [8], will be the starting point of our analysis.

The current investigation into optical trapping at low NA may also have some practical relevance, besides addressing a fundamental question on the nature of optical confining forces. A second motivation lies in the potential application of Optical Pickup Units (OPUs), known from optical data storage, for optical trapping. These OPUs are in fact miniature highprecision optical laboratories, consisting of a laser diode, collimating and beamshaping lenses, diffraction gratings, a segmented (quadrant) photo-detector, and a singlet aspherical objective lens of diffraction limited quality, equipped with focus and tracking actuation (see Figure 1). The bill of materials of OPUs is of the order 10 euro, so the key components of OPUs may be applied to construct compact cost-effective optical tweezers. Currently there are three formats available, each operating at a different wavelength and at a different NA: Compact Disc (CD $\lambda=785 \mathrm{~nm}, \mathrm{NA}=0.45)$, Digital Video Disc (DVD, $\lambda=655 \mathrm{~nm}, \mathrm{NA}=0.65)$, and Blu-ray Disc (BD, $\lambda=405 \mathrm{~nm}, \mathrm{NA}=0.85)$. Current OPUs usually offer compatibility between formats of some degree, i.e. they are capable of reading out multiple formats [9]-[15]. This means that it may be possible to combine an infrared laser diode of $C D$ with the NA $=0.85$ objective lens of $\mathrm{BD}$, which would constitute a reasonably efficient optical trap operating at a wavelength with limited photo-toxicity for biological specimens. This type of compact optical tweezers could possibly be retrofitted to conventional microscopes, in contrast to compact tweezers technologies targeting integrated microfluidic devices such as the dual beam fiber optical trap $[16,17]$ or the miniature micromirror array optical trap [18].

The paper is organized as follows. First we develop and analyze the theory of the axial trapping force (along the optical axis), then we proced with a short description and analysis of the lateral trapping force (perpendicular to the optical axis), and we conclude with an application of the theory to compact OPU-based optical tweezers.

\section{THE AXIAL TRAPPING FORCE}

\subsection{The limit of low NA}

We consider trapping of a sphere of radius a and refractive index $m \times n$ in a medium with refractive index $n$ ( $m$ is the re- 

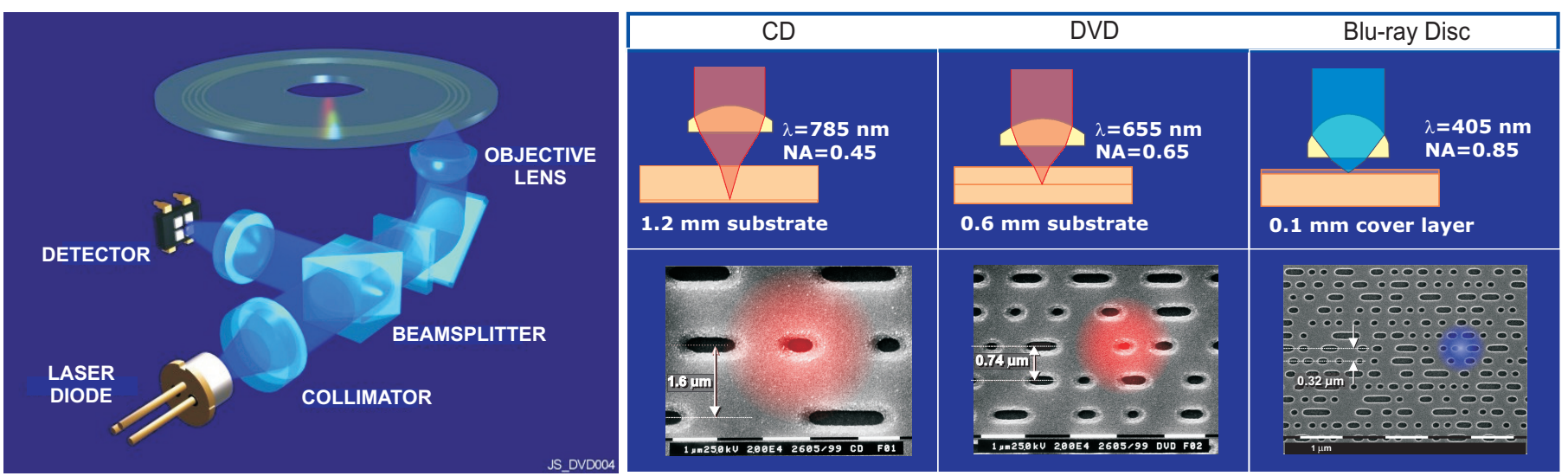

FIC. 1 Schematic drawing of an Optical Pickup Unit (left), and an overview of the three optical data storage formats (right). Images are courtesy of Jean Schleipen, Philips Research Laboratories.

fractive index contrast) by a focused beam of power $P$, numerical aperture NA and wavelength $\lambda$. Mazzoli, Maia Neto, and Nussenzveig have developed a generally applicable theory of the optical trapping force based on a multipole expansion of the incident and scattered field $[4,5]$. Approximations applicable to the Rayleigh-regime $(a \ll \lambda)$ and for the geometrical optics regime $(a \gg \lambda)$ can and have been derived from the general theory. Here we present analytical results pertaining to a different regime, namely the paraxial regime of small NA. A suitable expansion parameter is $\beta=\left(n-\sqrt{n^{2}-\mathrm{NA}^{2}}\right) / 2 n$. The regime of small $\beta$ appears to be surprisingly large. For example, for $\mathrm{NA}=0.85$ and focusing into a watery environment ( $n=1.33) \beta$ only takes the value 0.115 . Central in the theory of [4] are the quantities $G_{l}$ defined for any multipole order $l$. These $G_{l}$ are defined as integrals over the polar angle of incidence of the plane waves making up the focused incident field. In the paraxial regime the integrands may be considered constant, and an analytical evaluation of the integrals can be readily obtained. The approximation is justified for multipole orders $l$ such that $l^{2} \beta$ is small, as can be deduced from a Taylor-expansion of the integrands. From the theory of Miescattering it is known that Mie-coeffcients of multipole order $l$ only contribute significantly if $l<q$ with $q=2 \pi a / \lambda$ the reduced size parameter [19]. It follows that the approximation is quantitatively valid provided that $N A<2 / q$. This boils down to a bead diameter $2 a$ less than about the radius of the first dark Airy-ring surrounding the focal point $0.61 \lambda / N A$. For the small NA-values under consideration this is an extension of the Rayleigh-regime $a \ll \lambda$.

I have found the following expression for the axial force on the bead as a function of the position $z$ with respect to focus:

$$
\begin{aligned}
F= & \frac{n P}{c}\left[2 \beta V_{\mathrm{sc}}(m, q) \operatorname{sinc}(v)^{2}\right. \\
& \left.+\beta^{2} V_{\mathrm{gr}}(m, q) \frac{d}{d v}\left[\operatorname{sinc}(v)^{2}\right]\right],
\end{aligned}
$$

with the scaled axial position $v=2 \pi n \beta z / \lambda$, and with $\operatorname{sinc}(v)=\sin v / v$ the incident focused field along the optical axis, and where the functions $V_{\mathrm{sc}}(m, q)$ and $V_{\mathrm{gr}}(m, q)$ can be expressed in terms of the Mie-coefficients for the different multipole orders in scattering theory:

$$
\begin{aligned}
V_{\mathrm{sc}}(m, q)= & \operatorname{Re}\left[\sum_{l}(2 l+1)\left(a_{l}+b_{l}\right)-\right. \\
& -2 \sum_{l}\left\{\frac { l ( l + 2 ) } { l + 1 } \left(a_{l} a_{l+1}^{*}\right.\right. \\
& \left.\left.\left.+b_{l} b_{l+1}^{*}\right)+\frac{2 l+1}{l(l+1)} a_{l} b_{l}^{*}\right\}\right], \\
V_{\mathrm{gr}}(m, q)= & -\operatorname{Im}\left[\sum_{l}(2 l+1)\left(a_{l}+b_{l}\right)-\right. \\
& \left.-2 \sum_{l} l(l+2)\left(a_{l} a_{l+1}^{*}+b_{l} b_{l+1}^{*}\right)\right]
\end{aligned}
$$

Clearly, the axial force is the sum of a 'scattering' part, proportional to the intensity, and a 'gradient' part, proportional to the gradient of the intensity. The function $V_{\mathrm{sc}}(m, q)$ is up to a factor $(2 \pi / \lambda)^{2}$ equal to Debye's cross-section for calculating the force on a sphere illuminated by a plane wave [19], the other function $V_{\mathrm{gr}}(m, q)$ is novel. It may also be seen that both functions involve parts linear in the Mie-coefficients (the 'extinction' part, equal to the momentum taken from the incident wave per unit time) and parts quadratic in the Mie-coefficients (the 'scattering' part, equal to the momentum carried away by the scattered waves per unit time). In this sense, the adjective 'scattering' takes a double meaning!

\subsection{Dimensionless description of the trapping force}

The expression for the trapping force allows for a dimensionless analysis of trapping and the competition between the scattering and gradient forces by the introduction of a dimensionless trapping parameter:

$$
\eta=\frac{V_{\mathrm{sc}}(m, q)}{\beta V_{\mathrm{gr}}(m, q)},
$$

and a force constant:

$$
F_{0}=2 \frac{n P}{c} \beta^{3} \frac{V_{\mathrm{gr}}(m, q)^{2}}{V_{\mathrm{sc}}(m, q)} .
$$



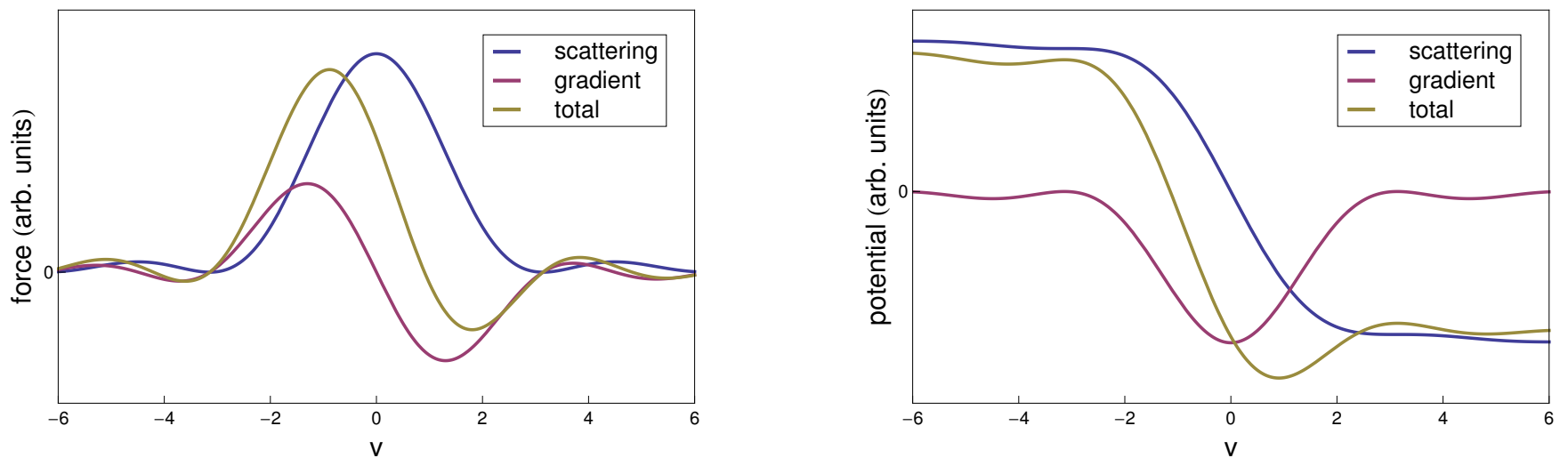

FIG. 2 Scaled axial force (left) and scaled potential (right) as a function of the reduced axial coordinate $v$ for $\eta=0.32$, at the optimum potential well depth (cf. sect. 2.4 ). In both graphs the 'scattering' contribution and the 'gradient' contribution is indicated.

Now the force may be expressed as:

$$
F=F_{0}\left[\eta^{2} \operatorname{sinc}(v)^{2}+\frac{\eta}{2} \frac{d}{d v}\left[\operatorname{sinc}(v)^{2}\right]\right] .
$$

The scaled force $F / F_{0}$ is thus a function of $v$ that depends only on the trapping parameter $\eta$. It appears that the trapping parameter $\eta$ determines the character of the trapping force. The gradient force dominates for small $\eta$, the scattering force dominates for large $\eta$. The gradient force stabilizes the trap, whereas the scattering force destabilizes the trap. Stable trapping thus only occurs when $\eta$ is sufficiently small, i.e. if NA is sufficiently high or if $a / \lambda$ is sufficiently small or if the refractive index contrast $m$ is sufficiently close to one. On the other hand, if $\eta$ is too small both forces are small. Both forces are even zero in the limit in which $\eta$ goes to zero. It follows that there must be an optimum value for $\eta$. Figure 2 shows the scattering force, gradient force and total force.

The force may be derived from a trapping potential:

$$
U=U_{0}\left[\eta^{2} \frac{1-\cos (2 v)-2 v \operatorname{Si}(2 v)}{2 v}-\frac{\eta}{2} \operatorname{sinc}(v)^{2}\right]
$$

where $\operatorname{Si}(v)$ is the so-called sine integral, and with:

$$
U_{0}=2 \frac{P}{\omega} \beta^{2} \frac{V_{\mathrm{gr}}(m, q)^{2}}{V_{\mathrm{sc}}(m, q)}
$$

where $\omega=2 \pi c / \lambda$ is the angular frequency. The scaled potential $U / U_{0}$ is thus a function of $v$ that depends only on the scaled size parameter $\eta$. Figure 2 shows the 'scattering'potential, 'gradient'-potential and total potential. Note that the total force cannot be expressed as the gradient of a single potential in case displacements of the bead in three dimensions are considered. In that case there is always a nonconservative component of the force.

\subsection{Dependence on refractive index contrast and bead size}

The dependence of the trapping parameter, force constant and energy constant on NA (via $\beta$ ) is straightforward, the dependence on $m$ and $q$ is implicit via the cross-sections $V_{\mathrm{gr}}(m, q)$ and $V_{\mathrm{sc}}(m, q)$. Figure 3 shows the dependence of the trapping parameter on $m$ and $q$. Clearly, $\eta$ increases with $m$, making the scattering force more dominant for a large refractive index contrast ratio. It appears that $\eta$ increases with $q$ for small $q$, reaches a maximum for values in the range 1-3 and then slowly decreases for large $q$. It follows that the scattering force dominates for intermediate $q$ values, roughly corresponding to bead diameters of the order of the wavelength inside the immersing medium. The dependence of the force and energy constants on $m$ and $q$ is proportional to $V_{\mathrm{gr}}(m, q)^{2} / V_{\mathrm{sc}}(m, q)$. This function is plotted in Figure 3 as well. It appears that there is little variation with $m$. For small $q$ this function is roughly constant (limiting value 3 for $q$ approaching zero), for $q>1$ it increases strongly with $q$.

In the Rayleigh-limit of small spheres $(a / \lambda \ll 1)$ in which the scattered field may be approximated as an electric dipole field (neglecting all higher order multipoles) simple analytical expressions for the trapping parameter, force constant, and energy constant can be obtained. It turns out that:

$$
\begin{aligned}
\eta & =\frac{2}{3 \beta} \frac{m^{2}-1}{m^{2}+2} q^{3} \\
F_{0} & =6 \beta^{3} \frac{n P}{c} \\
U_{0} & =6 \beta^{2} \frac{P}{\omega}
\end{aligned}
$$

i.e. the trapping parameter is proportional to the volume of the bead, making it a scaled size parameter, whereas the force and energy constants are independent of the refractive index contrast $m$ and the size parameter $q$.

\subsection{Equilibrium and potential well depth}

Equilibrium is defined by $F=0$, leading to:

$$
\operatorname{sinc}(v)=0
$$

which gives unstable equilibriums (potential maxima) at $v=\ldots,-2 \pi,-\pi,+\pi,+2 \pi, \ldots$ and:

$$
\eta \operatorname{sinc}(v)+\frac{d}{d v}[\operatorname{sinc}(v)]=0 .
$$

which gives stable equilibriums (potential minima). The relevant stable equilibrium satisfies $0<v_{\min }(\eta)<\pi$. The potential at this minimum is $U_{\min }(\eta)$. The equilibrium is stable provided the barrier to pass over to the unstable equilibrium 

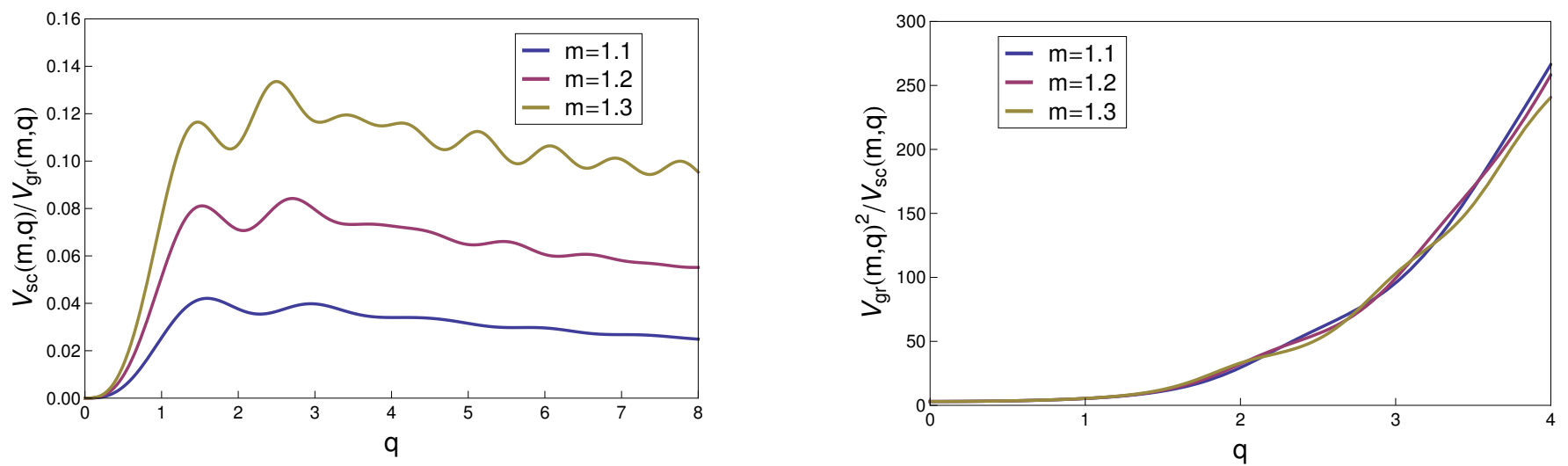

FIG. 3 The ratio of the scattering and gradient cross-sections $V_{\mathrm{sc}}(m, q) / V_{\mathrm{gr}}(m, q)=\beta \eta$ as a function of $q$ for $m=1.1, m=1.2$, and $m=1.3$ (top found in the Rayleigh-limit), and the force and energy constant dependence via $V_{\mathrm{gr}}(m, q)^{2} / V_{\mathrm{sc}}(m, q)$ as a function of $q$ for $m=1.1, m=1.2$, and $m=1.3$ (bottom). The cross-sections have been calculated by summing over the first 10 multipole contributions using Mathematica.

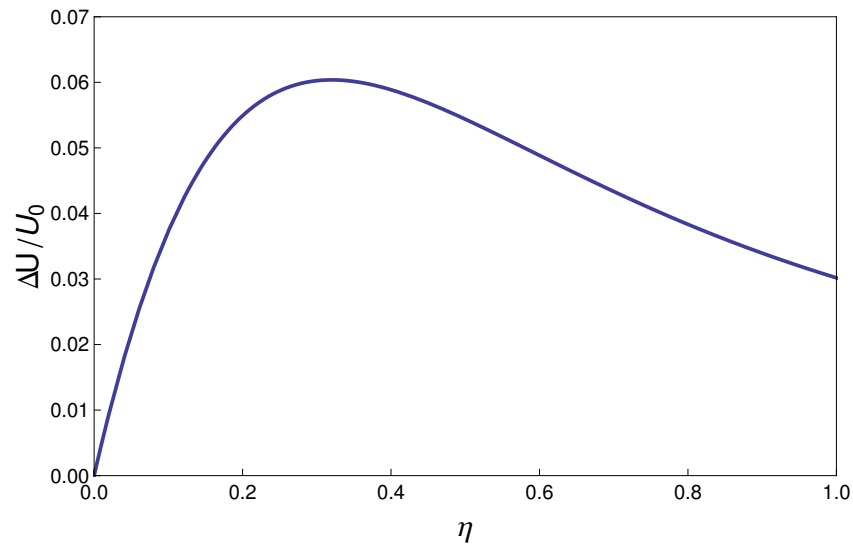

FIG. 4 The scaled potential well depth $\Delta U(\eta) / U_{0}$ as a function of the trapping parameter $\eta$.

at $v=\pi$ is much larger than the thermal energy $k_{\mathrm{B}} T$. The barrier energy or depth of the potential well is thus:

$$
\Delta U(\eta)=U(\pi)-U_{\min }(\eta)
$$

with

$$
U(\pi)=-\operatorname{Si}(2 \pi) \eta^{2} U_{0}=-1.41815 \eta^{2} U_{0}
$$

and the trapping is stable provided that $\Delta U(\eta) \gg k_{\mathrm{B}} T$. The scaled potential well depth $\Delta U(\eta) / U_{0}$ is a universal function that does not depend on any free parameter. This function is plotted in Figure 4. For small $\eta$ it may be approximated by the parabola $\eta / 2-\operatorname{Si}(2 \pi) \eta^{2}$. It can be determined numerically that the well depth is maximum at $\eta=\eta_{\text {opt }}=0.320$, and that the optimum is $U_{\mathrm{opt}}=0.06037 U_{0}$. This optimum point implies a relation between NA, $m$, and $q$. Figure 5 shows the dependence of $q_{\text {opt }}$ on $m$ for a range of NA-values. It appears that $q_{\text {opt }}$ decreases with increasing $m$, and increases with NA. The well-depth $U_{\text {opt }} \omega / P$ is also plotted in Figure 5. The optimum well depth decreases slighlty with $m$ and increases substantially with NA.

Analytical estimates for the optimum bead radius and well depth can be found in the Rayleigh-limit:

$$
\begin{aligned}
a_{\mathrm{opt}} & =\left(\frac{3 \beta \eta_{\mathrm{opt}}}{2}\right)^{1 / 3}\left(\frac{m^{2}-1}{m^{2}+2}\right)^{-1 / 3} \frac{\lambda}{2 \pi n} \\
& =0.783 \beta^{1 / 3}\left(\frac{m^{2}-1}{m^{2}+2}\right)^{-1 / 3} \frac{\lambda}{2 \pi n} \\
\Delta U_{\mathrm{opt}} & =0.362 \frac{\beta^{2} P}{\omega} .
\end{aligned}
$$

From the foregoing analysis it may be concluded that there is no fundamental lower limit below which there can be no stable optical trapping whatsoever. Provided the bead size and/or the refractive index contrast are small enough, the trapping parameter $\eta$ can always be brought in the range below about 0.5 , in which mechanically stable traps can be engineered. Thermodynamically stable traps further require a sufficiently high laser power. Of course, this does not imply that any bead with any arbitrary refractive index contrast can be trapped at these low NA values.

\subsection{Maximum forces and axial trap stiffness}

Another measure for the trap stability is the maximum force that is exerted on the bead when it is slightly displaced from it's equilibrium position (the point of largest gradient of the potential function). This force is larger for a deviation towards the incoming beam than away from the incoming beam due to the asymmetry of the potential well induced by the scattering force. For all but the smallest $\eta$ the asymmetry is substantial, leading to almost an order of magnitude difference between the force maxima on both sides of focus. Figure 6 shows the scaled force maxima on both sides of focus as a function of $\eta$. Apparently, there is an optimum in the force maximum on the side of focus away from the incoming beam. This optimum is at $\eta=0.52$, a slightly larger value for the trapping parameter than the value for optimum well depth. As a consequence, a good compromise between well depth and forces may be found for $\eta \approx 0.4$. At $\eta=0.32$ the force maxima on both sides of focus are $0.155 F_{0}$ and $0.0439 F_{0}$, respectively. If a symmet- 

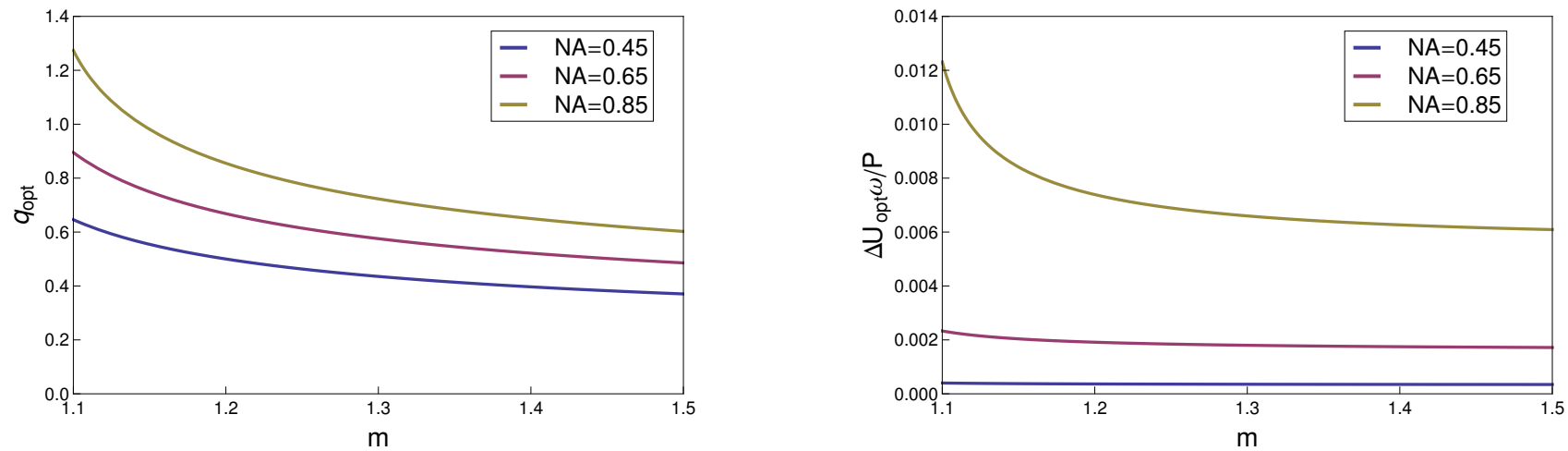

FIG. 5 Optimum $q_{\text {opt }}$ (top) and $U_{\mathrm{opt}} \omega / P$ (bottom) as a function of $m$ for NA $=0.45, \mathrm{NA}=0.65$, and $\mathrm{NA}=0.85$ and $n=1.33$. The data has been calculated using Mathematica using the first 8 multipole orders.
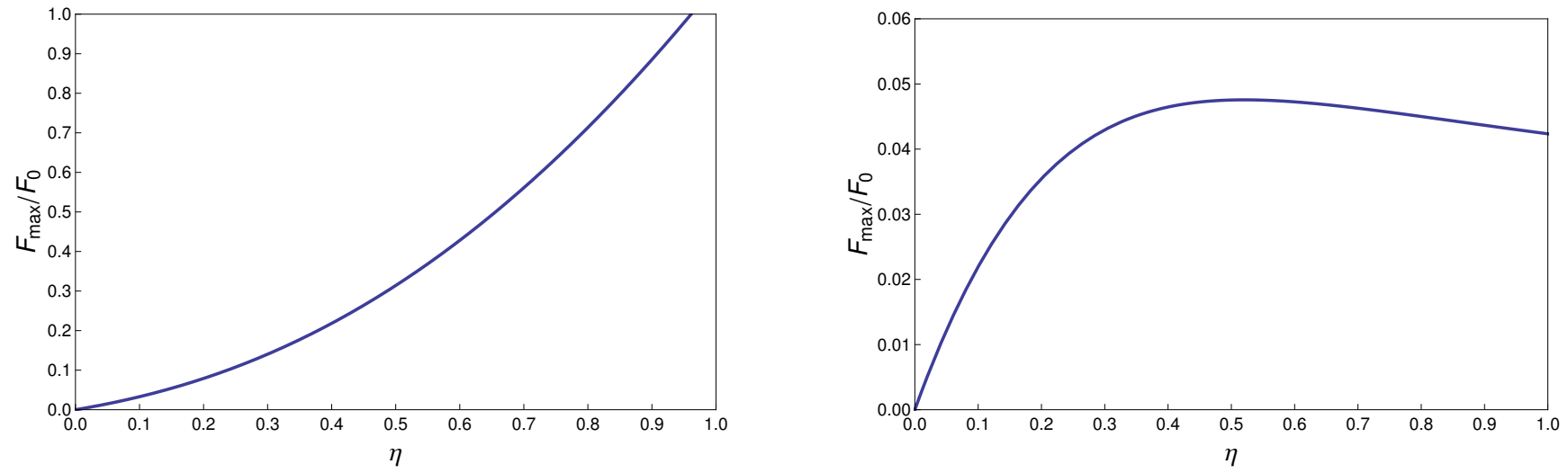

FIG. 6 Scaled maximum force $F_{\max } / F_{0}$ as a function of $\eta$ for $v<v_{\min }(\eta)$, towards the incoming beam (top), and for $v>v_{\min }(\eta)$, away from the incoming beam (bottom).

ric optical trap is required the trapping parameter should be restricted to values less than about 0.1 .

The trap stiffness is given by:

$$
\kappa=\kappa_{0}\left[\eta^{2} \frac{d \operatorname{sinc}(v)^{2}}{d v}+\frac{\eta}{2} \frac{d^{2} \operatorname{sinc}(v)^{2}}{d v^{2}}\right]_{v=v_{\min }(\eta)},
$$

where:

$$
\kappa_{0}=2 \frac{n^{2} P \omega}{c^{2}} \beta^{4} \frac{V_{\mathrm{gr}}(m, q)^{2}}{V_{\mathrm{sc}}(m, q)} \approx 6 \beta^{4} \frac{n^{2} P \omega}{c^{2}}
$$

where the approximation holds in the Rayleigh-limit. In thermal equilibrium the equipartition theorem gives that the mean square displacement is given by $\left\langle z^{2}\right\rangle=k_{\mathrm{B}} T / \kappa$. Figure 7 shows the scaled root mean square displacement $\left(\kappa / \kappa_{0}\right)^{-1 / 2}$ as a function of $\eta$. For the maximum well depth at $\eta=0.320$ we find $\left(\kappa / \kappa_{0}\right)^{-1 / 2}=3.23$. Compiling these data it follows that then:

$$
\sqrt{\left\langle z^{2}\right\rangle P}=0.794 \sqrt{\frac{k_{\mathrm{B}} T P}{\Delta U_{\mathrm{opt}}}} \frac{\lambda}{2 \pi n \beta} .
$$

This equation may serve to evaluate numerically the trap stiffness at optimum well depth conditions as a function of experimental parameters.

\subsection{Numerical results}

The universal curves for the barrier height $\Delta U / U_{0}$ and scaled maximum force $F_{\max } / F_{0}$ away from and towards focus may

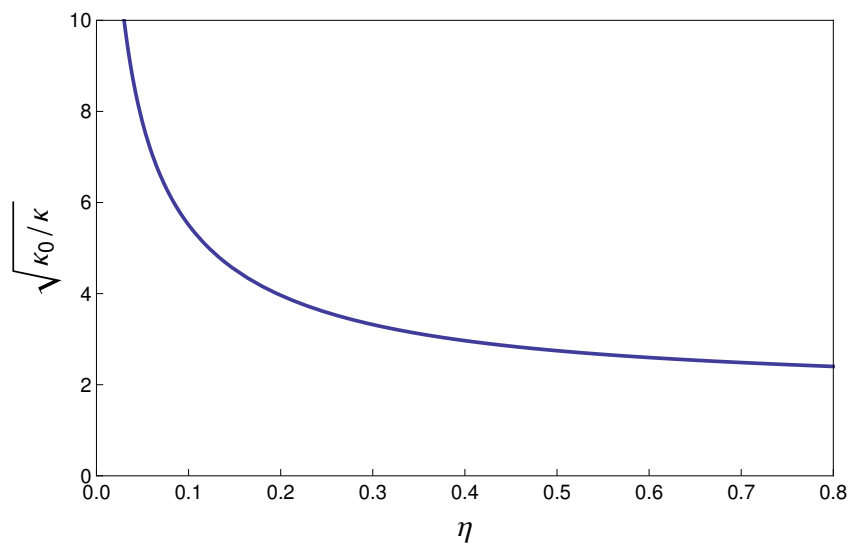

FIC. 7 Scaled mean square displacement in thermal equilibrium $\left(\kappa / \kappa_{0}\right)^{-1 / 2}$ as a function of $\eta$.

be compared with numerically calculated exact values, based on the theory of $[4,5]$. The trapping parameter $\eta$ is varied in these numerical calculations by varying the bead radius $a$. The results are shown in Figure 8 and Figure 9. The correspondence is quite well. Deviations occur for larger trapping parameters, in particular for high NA-values and values for the refractive index contrast $m$ close to one.

\section{THE LATERAL TRAPPING FORCE}

The lateral trapping forces are largely determined by the 'gradient'-force. By analogy with the expression for the 

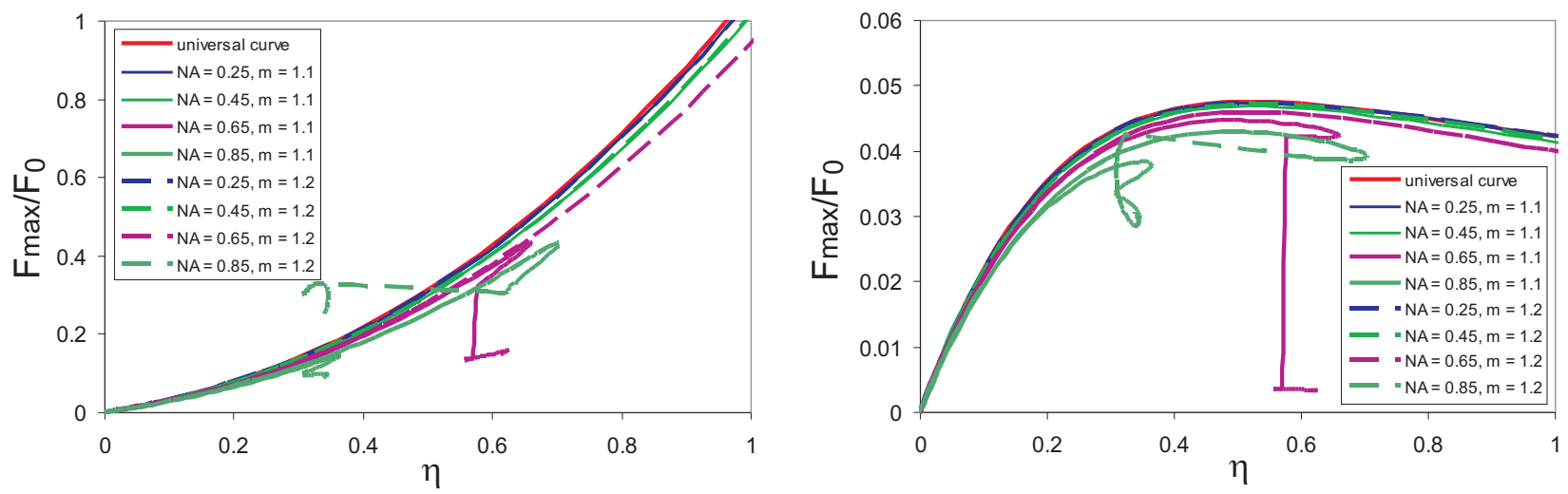

FIG. 8 Numerically calculated values for the scaled maximum forces towards (top) and away from (bottom) the incoming beam as a function of the scaled size parameter $\eta$ and the universal curve following from the analytical theory.

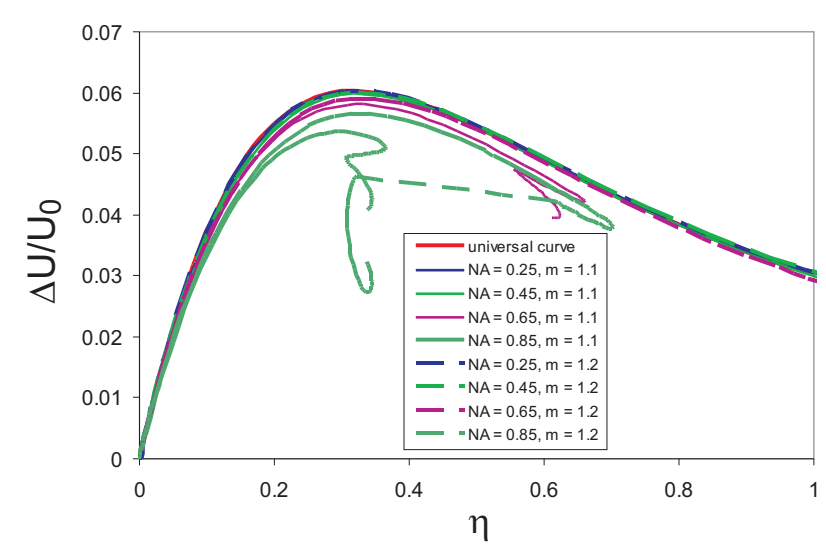

FIC. 9 Numerically calculated values for the scaled barrier height $\Delta U / U_{0}$ as a function of the trapping parameter $\eta$ and the universal curve following from the analytical theory.

'gradient'-force in the axial direction (which is proportional to the gradient of the intensity distribution) we may infer that the lateral trapping potential is given by the Airy-distribution $A(u)=2 J_{1}(u) / u$ :

$$
U=U_{0} A(u)^{2}
$$

with $u=2 \pi x \mathrm{NA} / \lambda$, and that the lateral trapping force is then given by:

$$
F=-\frac{\mathrm{NA}}{n \beta} \frac{d}{d u}\left[A(u)^{2}\right]
$$

It is assumed here that the bead is in the focal plane. It follows that the equations are strictly valid only if the trapping parameter $\eta$ is sufficiently small. Deviations in the axial position from the focal plane can be taken into account in a semi-quantitative manner by multiplying the potential and the force by a factor $\operatorname{sinc}^{2}\left(v_{\min }(\eta)\right)$. This is not fully exact as the intensity factorizes in the product of the axial function $\operatorname{sinc}^{2}(v)$ and the lateral function $A(u)^{2}$ only approximately. Nevertheless, this procedure may result in reasonable estimates.

The lateral trapping force is generally larger than the axial trapping force, as the former scales with a lower power of NA than the latter. The largest lateral force is found from:

$$
\frac{d^{2}}{d u^{2}}\left[A(u)^{2}\right]=0,
$$

which gives numerically $u=1.488$ for which:

$$
-\frac{d}{d u}\left[A(u)^{2}\right]=0.460 \text {. }
$$

For the condition of maximum well depth $\eta=0.320$ we have $v_{\min }(\eta)=0.906$. It follows that the maximum lateral trapping force is given by:

$$
F_{\text {lat,max }}=0.417 \frac{\mathrm{NA}}{n \beta} F_{0} .
$$

The lateral trap stiffness is given by:

$$
\begin{aligned}
\kappa_{\perp} & =\left(\frac{\mathrm{NA}}{n \beta}\right)^{2} \kappa_{0} \operatorname{sinc}^{2}\left(v_{\min }(\eta)\right) \frac{d^{2}}{d u^{2}}\left[A(u)^{2}\right]_{u=0} \\
& =0.453\left(\frac{\mathrm{NA}}{n \beta}\right)^{2} \kappa_{0} .
\end{aligned}
$$

The root mean square lateral displacement in thermal equilibrium can be expressed in terms of the root mean square axial displacement by:

$$
\sqrt{\left\langle x^{2}\right\rangle}=\sqrt{\frac{k_{\mathrm{B}} T}{\kappa_{\perp}}}=1.486 \frac{\mathrm{NA}}{n \beta} \sqrt{\left\langle z^{2}\right\rangle}
$$

This provides a simple scaling relation between the axial and lateral extension of the optical trap.

\section{DISCUSSION}

We can apply the analytical theory developed here to the three optical data storage formats $\mathrm{CD}(\lambda=785 \mathrm{~nm}, \mathrm{NA}=0.45)$, $\operatorname{DVD}(\lambda=655 \mathrm{~nm}, \mathrm{NA}=0.65)$, and $\operatorname{BD}(\lambda=405 \mathrm{~nm}$, $\mathrm{NA}=0.85)$. For these NA-values the paraxial parameter $\beta$ is equal to 0.029 (CD), 0.064 (DVD), and 0.115 (BD), for focusing into a medium with $n=1.33$. These values are sufficiently small compared to one to justify application of the present theory. Table 1 summarizes the main parameters: optimum bead radii in $\mathrm{nm}$, well depths in $k_{\mathrm{B}} T / W$, maximum forces in $\mathrm{pN} / \mathrm{W}$, and rms displacements in $\mathrm{nm} \sqrt{\mathrm{W}}$. The optimum bead radii happen to be in the range $60-80 \mathrm{~nm}$ for all 


\begin{tabular}{l|ccccccc} 
format & $\begin{array}{c}a_{\mathrm{opt}} \\
(\mathrm{nm})\end{array}$ & $\begin{array}{c}U_{\mathrm{opt}} / P \\
\left(k_{\mathrm{B}} T / W\right)\end{array}$ & $\begin{array}{c}F_{\mathrm{max},+} \\
(p N / W)\end{array}$ & $\begin{array}{c}F_{\text {max },-} \\
(p N / W)\end{array}$ & $\begin{array}{c}F_{\text {lat,max }} \\
(p N / W)\end{array}$ & $\begin{array}{c}z_{\mathrm{rms}} \sqrt{P} \\
(\mathrm{~nm} \sqrt{W})\end{array}$ & $\begin{array}{c}x_{\mathrm{rms}} \sqrt{P} \\
(\mathrm{~nm} \sqrt{W})\end{array}$ \\
\hline $\mathrm{CD}$ & 63 & 42 & 0.137 & 0.039 & 4.3 & 397 & 50.6 \\
$\mathrm{DVD}$ & 74 & 410 & 1.78 & 0.50 & 36.6 & 48.0 & 9.3 \\
$\mathrm{BD}$ & 70 & 849 & 21.0 & 5.8 & 314 & 16.5 & 4.4
\end{tabular}

TABLE 1 Calculated optimum bead radius, well depth, maximum forces, and rms axial displacement for numbers pertaining to the three optical data storage formats for silica beads in water $(m=1.45 / 1.33=1.1)$.

three formats. The well-depths are sufficiently large to ensure thermodynamic stability for moderate laser powers. Available laser powers in OPUs, at least in the ones with writing capability, are of the order $100 \mathrm{~mW}$. It appears that the forces increase drastically with increasing NA. Typical forces for CD, $\mathrm{DVD}$, and BD-based tweezers are in the range of $0.1 \mathrm{pN} / \mathrm{W}$, $1 \mathrm{pN} / \mathrm{W}$ and $10 \mathrm{pN} / \mathrm{W}$, respectively. The lateral forces appear to be one to two orders of magnitude larger than the axial forces. It may be concluded that CD based optical tweezers are likely too weak in practice, but BD based optical tweezers may appear to have some practical value. The experimental realization of the OPU-based compact optical tweezers is left for future explorations.

Another topic worthy of further study is the investigation of other approximation schemes than the paraxial treatment described in this paper. In particular the regime of small refractive index contrast may be of use for a simplified description of optical trapping of biological specimens. These objects, being composed of watery stuff in a watery environment, have an intrinsically small refractive index contrast. The theoretical machinery to study this limit is also available in the form of the (first order) Born approximation. The scattering problem can then be readily solved, and reasonably concise expressions for the trapping force and potential can possibly be found. It may be anticipated that in this limit only the gradient force survives, the scattering force being of higher order in the refractive index contrast.

Summarizing, I have presented a theory of optical trapping at low Numerical Aperture (NA). The theory provides an analytical and universal framework for describing potential well depth and forces. Key element is the dimensionless trapping parameter, which depends on the bead size to wavelength ratio, the refractive index contrast, and the NA, and which is a measure of the relative strength of the gradient and scattering forces. This single parameter description of optical trapping is suitable for bead sizes less than the diffraction limited spot size, which extends the Rayleigh-regime for the paraxial NAvalues under consideration.

\section{Acknowledgements}

Dirk Vossen is thanked for introducing me to the field of optical trapping, and for making the link between optical data storage and optical tweezers. Alfons van Blaaderen is acknowledged for providing the source code for the numerical analysis. The source code itself was written by Alexander Moroz.

\section{References}

[1] D. G. Grier, "A revolution in optical manipulation", Nature 424, 810-816 (2003).

[2] K. C. Neuman, and S. M. Block, "Optical trapping", Rev. Sci. Instrum. 75, 2787-2809 (2004).

[3] M. -T. Wei, and A. Chiou, "Three-dimensional tracking of Brownian motion of a particle trapped in optical tweezers with a pair of orthogonal tracking beams and the determination of the associated optical force constants", Opt. Express 13, 5798-5806 (2005).

[4] P. A. Maia Neto, and H. M. Nussenzveig, "Theory of optical tweezers", Europhys. Lett. 50, 702-708 (2000).

[5] A. Mazzoli, A. Maia Neto, and H. M. Nussenzveig, "Theory of trapping forces in optical tweezers", P. Roy. Soc. Lond. A 459, 30213041 (2003).

[6] J. P. Barton, D. R. Alexander, and S. A. Schaub, "Theoretical determination of net radiation force and torque for a spherical particle illuminated by a focused laser beam", J. Appl. Phys. 66, 4594-4602 (1989).

[7] $\emptyset$. Farsund, and B. U. Felderhof, "Force, torque, and absorbed energy for a body of arbitrary shape and constitution in an electromagnetic radiation field", Physica A 227, 108-130 (1996).

[8] N. B. Viana et al., "Absolute calibration of optical tweezers", Appl. Phys. Lett. 88, 131110 (2006).

[9] T. W. Tukker, and J. J. Vrehen, "Optical scanning device", W0/2006/077542 (2006).

[10] T. W. Tukker, and J. J. Vrehen, "Optical compensator, optical element, optical scanning head and optical scanning device", W0/2007/034389 (2007).

[11] T. Tanaka, K. Sano, K. Matsumura, H. Wada, K. Matsuzaki, K. Wakabayashi and Y. Komma, "Optical pickup for recording to duallayer $2 \mathrm{x}$-speed blu-ray disc, DVD and compact disc", Optical Data Storage Topical Meeting, 2006 , 240-242 (2006).

[12] T. Matsuo, T. Haruguchi, H. Takamure, S. Horinouchi, T. Koga, and S. Mizuno, "Optical pickup for BD/DVD/CD drive with $12.7 \mathrm{~mm}$ height", Optical Data Storage Topical Meeting, 2006, 261-263 (2006).

[13] M. Aiba, S. Kawakita, J. Sato, K. Kanno, and T. Yamada, "BD/DVD/CD compatible objective lens", Optical Data Storage Topical Meeting, 2006, 264-266 (2006).

[14] K. Yamazaki, H. Mori, T. Kawamura, Y. Kitada, and T. Kamisada, "Blu-ray disc/DVD compatible optical slim pickup for video camera drives", International Conference on Consumer Electronics, 2008, 9-13 (2008).

[15] K. Kaneda, T. Yasui, K. Takahashi, and Y. Asoma, "Development of a plastic objective Lens for BD/DVD/CD compatibility", Optical Data Storage Topical Meeting, 2009, 96-98 (2009).

[16] A. Constable, J. Kim, J. Mervis, F. Zarinetchi, and M. Prentiss, "Demonstration of a fiber-optical light-force trap", Opt. Lett. 18, 
1867-1869 (1993).

[17] S. J. Cran-McGreehin, K. Dholakia, and T. F. Krauss, "Monolothic integration of microfluidic channels and semiconductor lasers", Opt. Express 14, 7723-7729 (2006).

[18] F. Merenda, J. Rohner, J. -M. Fournier, and R. -P. Salathé, “Miniaturized high-NA focusing-mirror multiple optical tweezers", Opt. Express 15, 6075-6086 (2007).

[19] C. F. Bohren, and D. R. Huffman, Absorption and scattering of light by small particles (Wiley, New York, 1983). 